\title{
Oxytocin May be Superior to Gliptins as a Potential Treatment for Diabetic COVID-19 Patients
}

\author{
Phuoc-Tan Diep a* \\ ${ }^{a}$ MBChB, FRCPath-University Hospitals of Morecambe Bay, NHS Foundation Trust, United Kingdom. \\ Received 28 August 2020; Revised 15 October 2020; Accepted 29 December 2020; Published 16 January 2021
}

\begin{abstract}
Diabetes is a comorbidity associated with an increased risk of severe COVID-19. Gliptins are anti-diabetic drugs that inhibit dipeptidyl peptidase-4 (DPP4) and they have been proposed as a possible treatment for COVID-19 patients with and without diabetes due to their immunomodulatory properties. Oxytocin has also been proposed as a treatment for COVID-19 due to its immunomodulatory properties as well as other mechanisms. In addition, oxytocin has been identified as a natural DPP4 inhibitor. Therefore, oxytocin not only has the properties associated with DPP4 inhibition but it has numerous additional beneficial properties. It is proposed that oxytocin may be superior to DPP4 inhibitors for COVID-19 patients especially for patients with type 2 diabetes.
\end{abstract}

Keywords: Dipeptidyl Peptidase-4; DPP4 Inhibitor; Oxytocin; Diabetes; COVID-19; SARS-CoV-2.

Diabetes is a comorbidity associated with COVID-19. A number of researchers have proposed the use of dipeptidyl peptidase-4 (DPP4) inhibitors in diabetics with COVID-19 [1-4]. Gliptins are a class of anti-diabetic drugs comprised of peptides and small molecules that inhibit DPP4. DPP4 cleaves incretins such as glucagon-like peptide-1 (GLP-1). Therefore, inhibiting DPP4 results in a decreased breakdown of incretins. Incretins in turn increase insulin secretion and suppress glucagon which subsequently reduces blood glucose levels [3].

Gliptins have been proposed as a treatment for COVID-19 because DPP4 inhibition also modulates the immune system through inflammatory cytokine reduction, modification of macrophage activity and through the antiinflammatory properties of GLP-1 [1]. From an antiviral perspective, Middle East respiratory syndrome corona virus (MERS-CoV) enters cells via DPP4 and this is also a possible co-receptor for SARS-CoV-2 viral entry [2]. Therefore, inhibition could theoretically block entry of SARS-CoV-2. Also, through computational studies, three gliptins were identified that could possibly inhibit the SARS-CoV-2 main protease Mpro [4].

There is a natural endogenous DPP4 inhibitor, the cyclic nonapeptide neurohormone oxytocin. It was identified as a DPP4 inhibitor through computational studies and confirmed through in vitro studies [5]. Oxytocin has been proposed as an anti-diabetic drug in view of its other metabolic effects on adipose tissue, on the liver and on the pancreas [6]. It is reasonable, therefore, to postulate that oxytocin levels might be lower in type 2 diabetic patients than in non-diabetic patients. Indeed, at least one paper confirms this to be so [7].

Last but not least, there are papers that propose oxytocin as a defence or treatment for COVID-19 [8-11]. These papers identify numerous mechanisms that could treat COVID-19 including, but not limited to, immunomodulation of cytokines, neutrophils, lymphocytes, macrophages and supportive actions via nitric oxide. One of the papers also identifies lower oxytocin levels in susceptible groups [11]. Further, oxytocin not only has anti-diabetic and

* Corresponding author: ptdiep@doctors.org.uk

\section{doi http://dx.doi.org/10.28991/SciMedJ-2020-02-SI-10}

$>$ This is an open access article under the CC-BY license (https://creativecommons.org/licenses/by/4.0/).

(C) Authors retain all copyrights. 
immunomodulatory properties it also has cardioprotective and anabolic properties as well as having positive psychosocial functions [12].

In summary, oxytocin has potential anti-diabetic effects through DPP4 inhibition as well as through other mechanisms and oxytocin's proposed effects on COVID-19 potentially include inhibition of DPP4 in addition to the numerous mechanisms already identified. Therefore I propose that: (1) endogenous induction of oxytocin could increase resistance to COVID-19 and improve overall health; (2) exogenous oxytocin could be used as a treatment for COVID-19 especially in type 2 diabetics.

\section{Declaration of Competing Interest}

The authors declare that they have no known competing financial interests or personal relationships that could have appeared to influence the work reported in this paper.

\section{References}

[1] Solerte, S. B., Di Sabatino, A., Galli, M., \& Fiorina, P. (2020). Dipeptidyl peptidase-4 (DPP4) inhibition in COVID-19. Acta Diabetologica, 57(7), 779-783. doi:10.1007/s00592-020-01539-z.

[2] Bassendine, M. F., Bridge, S. H., McCaughan, G. W., \& Gorrell, M. D. (2020). COVID - 19 and comorbidities: A role for dipep-tidyl peptidase 4 (DPP4) in disease severity? Journal of Diabetes, 12(9), 649 - 658. doi:10.1111/1753-0407.13052.

[3] Iacobellis, G. (2020). COVID-19 and diabetes: Can DPP4 inhibition play a role? Diabetes Research and Clinical Practice, 162, 108125. doi:10.1016/j.diabres.2020.108125.

[4] Rao, P. P. N., Pham, A. T., Shakeri, A., Shatshat, A. E., Zhao, Y., Karuturi, R. C., \& Hefny, A. A. (2020). Drug repurposing: Dipeptidyl peptidase IV (DPP4) inhibitors as potential agents to treat SARS-CoV-2 (2019-nCov) infection. doi:10.21203/rs.3.rs-28134/v1.

[5] Chittepu, V. C. S. R., Kalhotra, P., Osorio-Gallardo, T., Jiménez-Martínez, C., Robles-de la Torre, R. R., Gallardo-Velazquez, T., \& Osorio-Revilla, G. (2019). New Molecular Insights into the Inhibition of Dipeptidyl Peptidase-4 by Natural Cyclic Peptide Oxyto-cin. Molecules, 24(21), 3887. doi:10.3390/molecules24213887.

[6] Elabd, S., \& Sabry, I. (2015). Two Birds with One Stone: Possible Dual-Role of Oxytocin in the Treatment of Diabetes and Oste-oporosis. Frontiers in Endocrinology, 6. doi:10.3389/fendo.2015.00121.

[7] Qian, W., Zhu, T., Tang, B., Yu, S., Hu, H., Sun, W., Pan, R., Wang, J., Wang, D., Yang, L., Mao, C., Zhou, L., \& Yuan, G. (2014). Decreased circulating levels of oxytocin in obesity and newly diagnosed type 2 diabetic patients. The Journal of clinical endo-crinology and metabolism, 99(12), 4683-4689. doi:10.1210/jc.2014-2206.

[8] Buemann, B., Marazziti, D., \& Uvnäs-Moberg, K. (2020). Can intravenous oxytocin infusion counteract hyperinflammation in COVID-19 infected patients? The World Journal of Biological Psychiatry, 1-30. doi:10.1080/15622975.2020.1814408.

[9] Imami, A. S., O'Donovan, S. M., Creeden, J. F., Wu, X., Eby, H., McCullumsmith, C. B., Uvnäs-Moberg, K., McCullumsmith, R. E., \& Andari, E. (2020). Oxytocin's Anti-inflammatory and Pro-immune functions in COVID-19: A Transcriptomic Signature Based Approach. Physiological genomics, 10.1152/physiolgenomics.00095.2020. Advance online publication. doi:10.1152/physiolgenomics.00095.2020.

[10] Soumier, A., \& Sirigu, A. (2020). Oxytocin as a potential defence against Covid-19? Medical Hypotheses, $140,109785$. doi:10.1016/j.mehy.2020.109785.

[11] Diep, P. T., Buemann, B., Uvnäs-Moberg, K., \& Marazziti, D. (2020). Oxytocin, a possible treatment for COVID-19? Everything to gain, nothing to lose. Clinical Neuropsychiatry, 17(3). doi:10.36131/CNEPUB20201703.

[12] Viero, C., Shibuya, I., Kitamura, N., Verkhratsky, A., Fujihara, H., Katoh, A., Ueta, Y., Zingg, H. H., Chvatal, A., Sykova, E., \& Dayanithi, G. (2010). REVIEW: Oxytocin: Crossing the bridge between basic science and pharmacotherapy. CNS neuroscience \& therapeutics, 16(5), e138-e156. doi:10.1111/j.1755-5949.2010.00185.x. 